\title{
Observations of prestellar cores: Probing the initial conditions for the IMF
}

\author{
Philippe André ${ }^{1}$ \\ ${ }^{1}$ CEA Saclay, DSM/DAPNIA, Service d'Astrophysique, F-91191 Gif-sur-Yvette Cedex, France \\ email: pandre@cea.fr
}

\begin{abstract}
Several (sub)millimeter-wave studies of nearby star-forming regions have revealed self-gravitating prestellar condensations that seem to be the direct progenitors of individual stars and whose mass distribution resembles the IMF. In a number of cases, small internal and relative motions have been measured for these condensations, indicating they are much less turbulent than their parent cloud and do not have time to interact before evolving into protostars and pre-main sequence stars. These findings suggest that the IMF is at least partly determined by pre-collapse cloud fragmentation and that one of the keys to understanding the origin of stellar masses lies in the physical mechanisms responsible for the formation and decoupling of prestellar cores within molecular clouds.
\end{abstract}

Keywords. stars: formation, ISM: clouds, ISM: structure

\section{Introduction}

One of the main limitations in our present understanding of the star formation process is that we do not know well the initial stages of cloud fragmentation and collapse into protostars. In particular, there is a major ongoing controversy between two schools of thought for the formation and evolution of dense cores within molecular clouds: The classical picture based on magnetic support and ambipolar diffusion (e.g. Shu et al. 1987, 2004; Mouschovias \& Ciolek 1999) has been seriously challenged by a new, more dynamic picture, which emphasizes the role of supersonic turbulence in supporting clouds on large scales and generating density fluctuations on small scales (e.g. Padoan \& Nordlund 2002, Mac Low \& Klessen 2004).

Improving our knowledge of the initial stages of star formation is crucially important since there is now good evidence that these stages control the origin of the stellar initial mass function (IMF). Indeed, observations suggest that the effective reservoirs of mass required for the formation of individual stars are already selected at the prestellar core stage. First, detailed (sub)-millimeter emission and infrared absorption mapping of a few nearby sources indicates that the density profiles of prestellar cores typically feature flat inner regions and sharp outer edges, hence are reminiscent of the density structure expected for finite-size/mass, self-gravitating isothermal spheroids (such as 'Bonnor-Ebert' spheres) (e.g. Ward-Thompson et al. 1994, Bacmann et al. 2000, Alves et al. 2001). Second, several ground-based (sub)-millimeter continuum surveys of nearby, compact cluster-forming clouds have uncovered 'complete' (but small) samples of prestellar condensations whose mass distributions resemble the stellar IMF (see $\S 2$ ).

\section{Link between the prestellar core mass distribution and the IMF}

Wide-field (sub)mm dust continuum mapping is a powerful tool to take a census of dense cores within star-forming clouds. The advent of large-format bolometer arrays on 


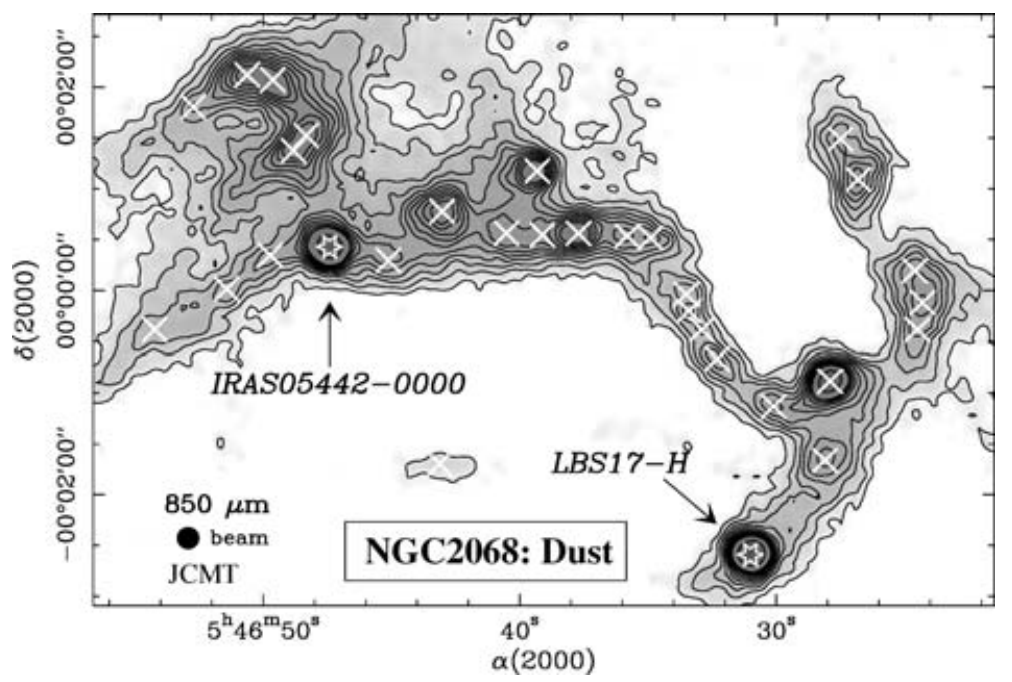

Figure 1. SCUBA $850 \mu \mathrm{m}$ dust continuum map of the NGC 2068 protocluster extracted from the mosaic of NGC 2068/2071 by Motte et al. (2001). A total of 30 compact starless condensations (marked by crosses), with masses between $\sim 0.4 M_{\odot}$ and $\sim 4.5 M_{\odot}$, are detected in this $\sim 1 \mathrm{pc} \times$ 0.7 pc field.

(sub)millimeter radiotelescopes such as the IRAM 30m and the JCMT has led to the identification of numerous cold, compact condensations that do not obey the Larson (1981) self-similar scaling laws of molecular clouds and are intermediate in their properties between diffuse CO clumps and infrared young stellar objects (cf. André et al. 2000 and Ward-Thompson et al. 2006 for reviews). As an example, Fig. 1 shows the condensations found by Motte et al. (2001) at $850 \mu \mathrm{m}$ in the NGC 2068 protocluster (Orion B). Such highly concentrated (sub)millimeter continuum condensations are at least 3 to 6 orders of magnitude denser than typical CO clumps (e.g. Kramer et al. 1998) and feature large (> > 50\%) mean column density contrasts over their parent background clouds, strongly suggesting they are self-gravitating. The latter is directly confirmed by line observations in a number of cases (see $\S 3.1$ below). A small fraction of these condensations lie at the base of powerful jet-like outflows and correspond to very young protostars which have not yet accreted the majority of their final masses (Class 0 objects - cf. André et al. 2000). However, the majority of them are starless/jetless and appear to be the immediate prestellar progenitors of individual protostars or protostellar systems.

In particular, the mass distribution of these prestellar condensations is remarkably similar to the stellar IMF (e.g. Motte, André, Neri 1998 - hereafter MAN98). This is illustrated in Fig. 2 which shows the cumulative mass spectrum of the 57 starless condensations found by MAN98 in their $1.2 \mathrm{~mm}$ continuum survey of the $\rho$ Ophiuchi main cloud with the IRAM $30 \mathrm{~m}$ telescope. These condensations, which were identified using a multi-resolution wavelet analysis (cf. Starck et al. 1998), are seen on the same spatial scales as protostellar envelopes (i.e., $\sim 2500-5000 \mathrm{AU}$ or $\sim 15^{\prime \prime}-30^{\prime \prime}$ in $\rho \mathrm{Oph}$ ). Their mass spectrum is consistent with the Salpeter power-law IMF at the high-mass end and shows a tentative flattening below $\sim 0.4 M_{\odot}$ (see Fig. 2). The latter is reminiscent of the break observed in the IMF of field stars at $\sim 0.5 M_{\odot}$ (e.g. Kroupa 2001, Chabrier 2003), also present in the mass function of $\rho$ Oph pre-main sequence objects (Bontemps et al. 2001 - see star symbols in Fig. 2). If real, this flattening occurs at a mass comparable 


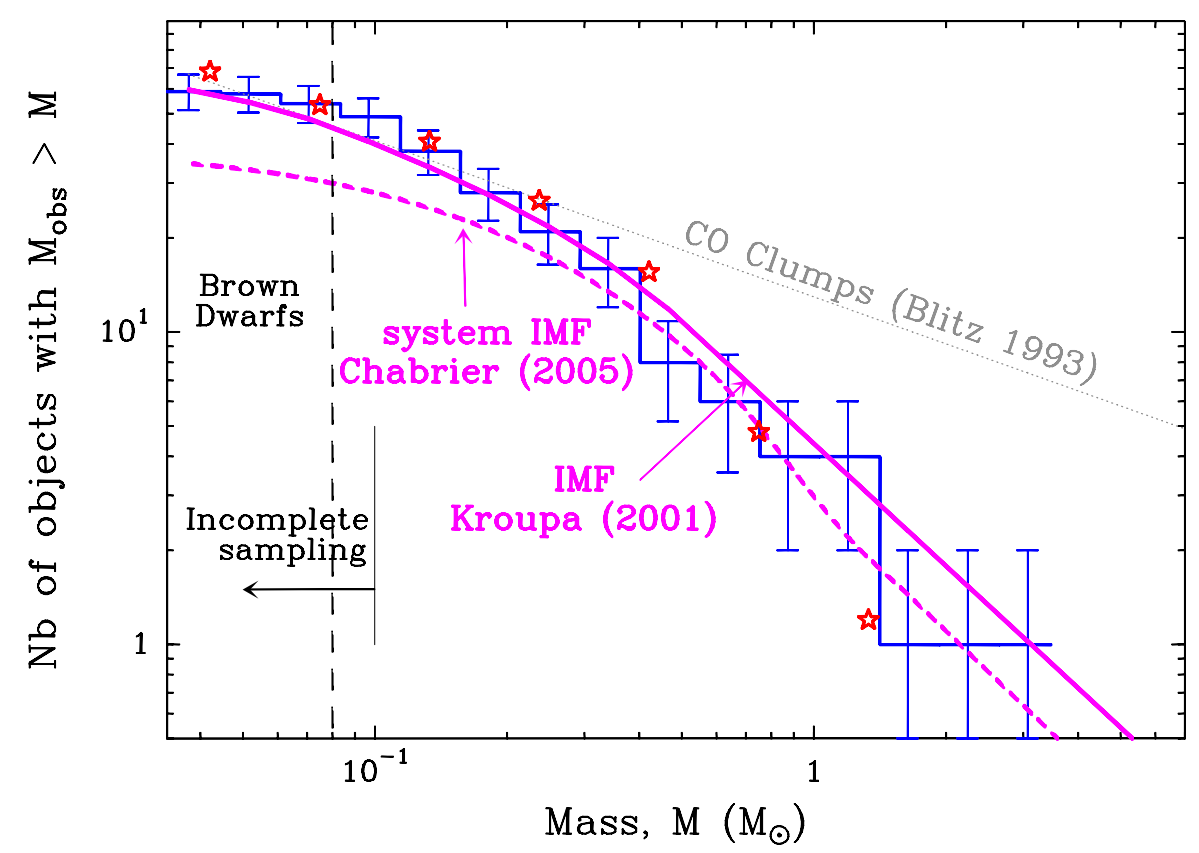

Figure 2. Cumulative mass distribution of a sample of 57 prestellar condensations, complete down to $\sim 0.1 M_{\odot}$, in the $\rho$ Oph protocluster (histogram with error bars - from MAN98). For comparison, the solid curve shows the shape of the field star IMF (e.g. Kroupa 2001), while the dashed curve corresponds to the IMF of multiple systems (e.g. Chabrier 2005). The star markers represent the mass function of $\rho$ Oph (primary) pre-main sequence objects as derived from a mid-IR survey with ISOCAM (Bontemps et al. 2001). The dotted line shows a $N(>M) \propto M^{-0.6}$ power-law distribution corresponding to the typical mass spectrum found for CO clumps - see Blitz 1993 and Kramer et al. 1998).

to the typical Jeans mass in the dense $\left(n_{\mathrm{H} 2} \sim 10^{5} \mathrm{~cm}^{-3}\right) \mathrm{DCO}^{+}$cores of the $\rho$ Oph cloud (cf. Loren et al. 1990). Interestingly, the observed mean separation between $\rho$ Oph condensations $(\sim 6000 \mathrm{AU})$ is also consistent with the Jeans length at the same density.

Such a close resemblance of their mass spectrum to the IMF in both shape and mass scale suggests that the starless condensations identified in the (sub)millimeter dust continuum are about to form stars on a one-to-one basis, with a high local efficiency, i.e., $M_{\star} / M_{\text {pre }} \gtrsim 50 \%$. This strongly supports scenarios according to which the bulk of the IMF is at least partly determined by pre-collapse cloud fragmentation (e.g. Larson 1985, 2005; Elmegreen 1997; Padoan \& Nordlund 2002).

The results of MAN98 in $\rho$ Oph have been essentially confirmed by independent $850 \mu \mathrm{m}$ SCUBA and $1.2 \mathrm{~mm}$ SIMBA surveys of the same region with JCMT and SEST (Johnstone et al. 2000, and Stanke et al. 2006, respectively). Similar results have also been found in other nearby cluster-forming clouds such as Serpens (Testi \& Sargent 1998), Orion B (Motte et al. 2001), and Perseus (Enoch et al. 2006). Furthermore, in a recent near-IR extinction imaging study of the Pipe dark cloud complex, Alves et al. (2006) also found a dense core mass function closely following the shape of the IMF.

Appealing as a direct connection between the prestellar core mass distribution (CMD) and the IMF might be, several caveats should be kept in mind. First, although core mass estimates based on optically thin (sub)millimeter dust continuum emission are 
straightforward, they rely on uncertain assumptions about the dust (temperature and emissivity) properties. Second, current determinations of the CMD are limited by smallnumber statistics in any given cloud and may be affected by incompleteness at the lowmass end. With Herschel, the future submillimeter space telescope to be launched by ESA in 2008, it will be possible to dramatically improve on the statistics and to largely eliminate the mass uncertainties through direct measurements of the dust temperatures (cf. André \& Saraceno 2005). Last but not least, the shape of the CMD agrees better with the IMF of individual field stars (solid curve in Fig. 2) than with the IMF of multiple systems (dashed curve in Fig. 2). This is surprising since current surveys for prestellar cores do not have enough spatial resolution to probe core multiplicity. Furthermore, multiple systems are believed to form after the prestellar stage by subsequent dynamical fragmentation during the collapse phase, close to the time of protostar formation (e.g. Goodwin et al. 2006). Thus, one would expect the masses of prestellar cores to be more directly related to the masses of multiple systems than to the masses of individual stars. It is possible that a fraction at least of the cores observed below $\sim 0.2-0.3 M_{\odot}$ (cf. Fig. 2) are not gravitationally bound, hence not prestellar in nature (but see $\S 3.1$ below). This also implies that other processes besides pure cloud fragmentation are not ruled out and may play an important additional role in generating the low-mass $\left(M<0.3 M_{\odot}\right)$ end of the IMF (cf. Bate et al. 2003, Ballesteros-Paredes et al. 2006).

\section{Kinematics of protocluster condensations}

Investigating the dynamical properties of the prestellar condensations identified in submillimeter dust continuum surveys is of great interest to discriminate between possible theoretical scenarios for core formation and evolution. Interesting results have emerged from recent molecular line studies using tracers such as $\mathrm{NH}_{3}, \mathrm{~N}_{2} \mathrm{H}^{+}, \mathrm{N}_{2} \mathrm{D}^{+}$, and $\mathrm{DCO}^{+}$, which do not deplete onto dust grains until fairly high densities (e.g. Tafalla et al. 2002, Crapsi et al. 2005).

\subsection{Internal motions}

The small-scale $(\sim 0.03 \mathrm{pc})$ prestellar condensations observed in the Ophiuchus, Serpens, Perseus, and Orion cluster-forming regions (cf. Fig. 1) are characterized by fairly narrow $\left(\Delta V_{F W H M} \lesssim 0.5 \mathrm{~km} \mathrm{~s}^{-1}\right)$ line widths in optically thin tracers of dense gas such as $\mathrm{N}_{2} \mathrm{H}^{+}$(1-0) (see, e.g., Fig. 3 and Belloche et al. 2001). For instance, the typical nonthermal velocity dispersion observed toward the starless condensations of the $\rho$ Oph protocluster is about half the thermal velocity dispersion of $\mathrm{H}_{2}\left(\sigma_{N T} / \sigma_{T} \sim 0.7\right.$ - Belloche et al. 2001). This indicates subsonic or at most transonic levels of internal turbulence and suggests that the initial conditions for individual protostellar collapse are relatively free of turbulence $\left(\sigma_{N T}<\sigma_{T} \sim 0.2 \mathrm{~km} \mathrm{~s}^{-1}\right)$, even when the parent cluster-forming clouds/cores have supersonic levels of turbulence $\left(\sigma_{N T} \gtrsim 0.4 \mathrm{~km} \mathrm{~s}^{-1}-\right.$ cf. Loren et al. 1990; Jijina et al. 1999). These findings are in qualitative agreement with the 'kernel' picture proposed by Myers (1998), according to which protocluster condensations correspond to zones of minimum turbulence, of size comparable to the cutoff wavelength for MHD waves, developing in turbulent cloud cores.

Importantly, the narrow linewidths measured in $\mathrm{N}_{2} \mathrm{H}^{+}(1-0)$ imply virial masses which generally agree within a factor of $\sim 2$ with the mass estimates derived from the dust continuum. This confirms that most of the starless condensations identified in the (sub)mm continuum are self-gravitating and very likely prestellar in nature. For instance, based on comprehensive $\mathrm{N}_{2} \mathrm{H}^{+}$(1-0) observations of the $\rho$ Oph protocluster, André et al. (2006) conclude that more than $\sim 70 \%$ of the starless condensations found by MAN98 at $1.2 \mathrm{~mm}$, 


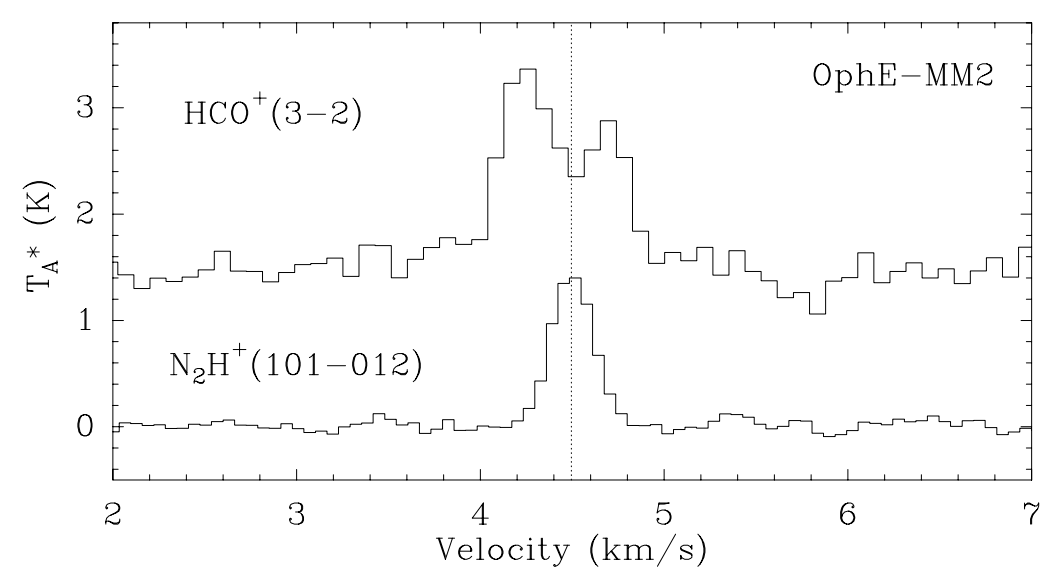

Figure 3. $\mathrm{HCO}^{+}(3-2)$ and $\mathrm{N}_{2} \mathrm{H}^{+}(101-012)$ spectra observed at the IRAM $30 \mathrm{~m}$ telescope toward the starless $1.2 \mathrm{~mm}$ continuum condensation E-MM2 identified by MAN98 in the $\rho$ Oph protocluster. The optically thick $\mathrm{HCO}^{+}$line is self-absorbed and skewed to the blue, which is the classical signature of collapse motions (e.g. Evans 1999), while the optically thin $\mathrm{N}_{2} \mathrm{H}^{+}$line is narrow $\left(\Delta V \lesssim 0.3 \mathrm{~km} \mathrm{~s}^{-1}\right)$ indicating a small level of turbulence. (From Belloche et al. 2001.)

and essentially all of those more massive than $\sim 0.1 M_{\odot}$, are gravitationally bound. The status of the $\rho$ Oph condensations less massive than $\sim 0.1 M_{\odot}$ is less clear since these are weaker and often undetected in $\mathrm{N}_{2} \mathrm{H}^{+}$. Some of them may possibly be unbound transient objects (see also end of $\S 2$ above).

The notion that a majority of the starless condensations identified in the (sub)millimeter dust continuum are prestellar and on the verge of forming protostars is further supported by the detection of infall motions toward some of them. As an example, toward OphE-MM2 in the $\rho$ Oph protocluster, the optically thick $\mathrm{HCO}^{+}(3-2)$ line exhibits a self-absorbed, double-peaked profile with a blue peak stronger than the red peak, while low optical depth lines such as $\mathrm{N}_{2} \mathrm{H}^{+}(101-012)$ are single-peaked and peak in the absorption dip of $\mathrm{HCO}^{+}(3-2)$ (see Fig. 3). This type of blue asymmetry in optically thick line tracers is now accepted as a classical spectroscopic signature of collapse (cf. Evans 1999). The infall speeds derived from radiative transfer modeling are $\sim 0.1-0.3 \mathrm{~km} \mathrm{~s}^{-1}$ (e.g. Belloche et al. 2001), consistent with a typical condensation lifetime $\sim 10^{5} \mathrm{yr}$.

\subsection{Relative motions}

Line observations can also provide information on the relative motions between condensations, as well as on possible global, large-scale motions in the parent protoclusters.

For instance, André et al. (2006) have analyzed the distribution of line-of-sight velocities observed toward 45 condensations of the $\rho$ Oph protocluster (see also Belloche et al. 2001). The results indicate a global, one-dimensional velocity dispersion $\sigma_{1 D} \sim 0.37 \mathrm{~km}$ $\mathrm{s}^{-1}$ about the $\rho$ Oph mean systemic velocity. Assuming isotropic random motions, this corresponds to a three-dimensional core-core velocity dispersion $\sigma_{3 D} \sim 0.64 \mathrm{~km} \mathrm{~s}{ }^{-1}$. With a $\rho$ Oph central cloud diameter of $\sim 1.1 \mathrm{pc}$, such a small velocity dispersion implies a typical crossing time $D / \sigma_{3 D} \sim 1.7 \times 10^{6} \mathrm{yr}$. The crossing times determined for the individual $\mathrm{DCO}^{+}$cores or subclusters of $\rho$ Oph are only slightly shorter $\left(\sim 0.6 \times 10^{6} \mathrm{yr}\right)$. 
Since neither the age of the embedded IR cluster nor the lifetime of the $1.2 \mathrm{~mm}$ condensations can be much larger than $10^{6}$ yr (cf. Bontemps et al. 2001 and $\S 3.1$ above), it appears that the $\rho$ Oph pre-stellar condensations do not have time to orbit through the protocluster gas and collide with one another (even inside individual subclusters) before evolving into pre-main sequence young stars (André et al. 2006). (This would require several crossing times - cf. Elmegreen 2001.) Similar results have been obtained for 25 condensations in the NGC 2068 protocluster (Belloche et al. in prep.).

While dynamical interactions between protostellar condensations may sometimes occur at the very center of massive, collapsing protoclusters (cf. Peretto et al. 2006), the results obtained in $\rho$ Oph and NGC 2068 seem inconsistent with models which resort to interactions and competitive accretion to build up the bulk of the stellar IMF at the protostellar stage (e.g. Bate et al. 2003). As the estimated tidal-lobe radius of the $\rho$ Oph condensations is comparable to their observed radius $\lesssim 5000$ AU (cf. MAN98), competitive accretion at the prestellar stage may nevertheless play a role in limiting the condensation masses (see Bonnell et al. 2001).

\section{Conclusions and future prospects}

The mass distribution of prestellar cores in star-forming clouds appears to be consistent with the stellar IMF between $\sim 0.1 M_{\odot}$ and $\sim 5 M_{\odot}$, although large uncertainties remain especially at the low- and high-mass ends (cf. $\S 2$ ). Small internal and relative motions are measured for these prestellar cores, implying that they are much less turbulent than their parent cloud and generally do not have time to interact before collapsing to (proto)stars (cf. $\S 3$ ). Taken at face value, these results are at variance with models in which dynamical interactions and competitive accretion play a key role in shaping the distribution of stellar masses (e.g. Bate et al. 2003). They strongly support scenarios according to which the IMF is largely determined at the prestellar stage and individual protostellar collapse is initiated in decoupled self-gravitating condensations resulting from turbulent (e.g. Padoan \& Nordlund 2002) and/or magnetic (e.g. Shu et al. 2004) cloud fragmentation.

In cluster-forming clouds like $\rho$ Oph, the star formation efficiency within each condensation is high (cf. $\S 2)$ : most $(>50 \%)$ of the initial mass at the onset of collapse seems to end up in a star (or a stellar system). There is some evidence that cluster-forming clumps are in a state of global collapse induced by external triggers (e.g. Peretto et al. 2006, Nutter et al. 2006). A promising mechanism in this case is purely gravitational, Jeans-like fragmentation of compressed cloud layers or thin expanding shells, a process which can produce a fragment mass spectrum approaching the Salpeter IMF (Palouš et al. 2003; Palouš, this volume).

In regions of more distributed star formation such as Taurus or the Pipe Nebula, the size of individual cores is larger (e.g. MAN98), the local star formation efficiency is lower ( 15\%-30\% - Onishi et al. 2002, Alves et al. 2006), and the feedback of protostellar outflows may be more important in limiting accretion and defining stellar masses (e.g. Shu et al. 2004).

To fully understand how the IMF comes about, it is crucial to further investigate the processes by which prestellar cores form and evolve in molecular clouds. With present submillimeter instrumentation, observational studies are limited by small-number statistics and restricted to the nearest regions. The advent of major new facilities at the end of the present decade should yield several breakthroughs in this field. With an angular resolution at 75-300 $\mu \mathrm{m}$ comparable to, or better than, the largest ground-based millimeter-wave radiotelescopes, Herschel, the Far InfraRed and Submillimeter Telescope to be launched by ESA in 2008 (cf. Pilbratt 2005), will make possible complete 
surveys for prestellar cores down to the proto-brown dwarf regime in the cloud complexes of the Gould Belt (cf. André \& Saraceno 2005). High-resolution $\left(0.01^{\prime \prime}-0.1^{\prime \prime}\right)$ imaging with the 'Atacama Large Millimeter Array' (ALMA, becoming partly available around 2009 , fully operational in 2013 - cf. Wootten 2001) at $\sim 450 \mu \mathrm{m}-3 \mathrm{~mm}$ will beat source confusion and allow us to probe individual condensations in distant, massive protoclusters, all the way to the Galactic center and the Magellanic clouds. Complementing each other nicely, Herschel and ALMA will tremendously improve our global understanding of the initial stages of star formation in the Galaxy.

\section{References}

Alves, J.F., Lada, C.J. \& Lada, E.A. 2001, Nature 409, 159

Alves, J.F., Lombardi, M. \& Lada, C.J. 2006, submitted

André, Ph., Belloche, A., Motte, F. \& Peretto, N. 2006, in preparation

André, Ph. \& Saraceno, P. 2005, in: A. Wilson (ed.), The Dusty and Molecular Universe: A Prelude to Herschel and ALMA (ESA SP), 577, p. 179

André, P., Ward-Thompson, D. \& Barsony, M. 2000, in: V. Mannings, A.P. Boss \& S.S. Russell (eds.), Protostars and Planets IV (Tucson: Univ. of Arizona), p. 59

Bacmann, A., André, P., Puget, J.-L., Abergel, A., Bontemps, S. \& Ward-Thompson, D. 2000, $A \mathscr{E A} 361,555$

Ballesteros-Paredes, J., Gazol, A., Kim, J., Klessen, R.S. et al. 2006, ApJ 637, 384

Bate, M.R., Bonnell, I.A. \& Bromm, V. 2003, MNRAS 339, 577

Belloche, A., André, P. \& Motte, F. 2001, in: T. Montmerle \& P. André (eds.), From Darkness to Light (ASP-CS), 243, p. 313

Blitz, L. 1993, in: E.H. Levy \& J.I. Lunine (eds.), Protostars \& Planets III (Tucson: Univ. of Arizona), p. 125

Bonnell, I.A., Bate, M. R., Clarke, C. J. \& Pringle, J.E. 2001, MNRAS 323, 785

Bontemps, S., André, P., Kaas, A.A. et al. 2001, A\&A 372, 173

Chabrier, G. 2003, ApJ 586, L133

Chabrier, G. 2005, in: E. Corbelli et al. (eds.), The Initial Mass Function 50 years later (Dordrecht: Springer), p.41

Crapsi, A., Caselli, P., Walmsley, C.M., Myers, P.C. et al. 2005, ApJ 619, 379

Elmegreen, B. 1997, ApJ 486, 944

Elmegreen, B. 2001, in: T. Montmerle \& P. André (eds.), From Darkness to Light (ASP-CS), 243 , p. 255

Enoch, M.L., Young, K.E., Glenn, J., Evans, N.J. et al. 2006, ApJ 638, 293

Evans, N.J. 1999, ARAA 37, 311

Goodwin, S.P., Kroupa, P., Goodman, A. \& Burkert, A. 2006, in: B. Reipurth, D. Jewitt, K. Keil (eds.), Protostars $\&$ Planets $V$, in press

Jijina, J., Myers, P.C. \& Adams, F.C. 1999, ApJS 125, 161

Johnstone, D., Wilson, C. D., Moriarty-Schieven, G., et al. 2000, ApJ 545, 327

Kramer, C., Stutzki, J., Rohrig, R. \& Corneliussen, U. 1998, A\& A 329, 249

Kroupa, P. 2001, MNRAS 322, 231

Larson, R.B. 1981, MNRAS 194, 809

Larson, R.B. 1985, MNRAS 214, 379

Larson, R.B. 2005, MNRAS 359, 211

Loren, R.B., Wootten, A. \& Wilking, B.A. 1990, ApJ 365, 229

Mac Low, M.-M. \& Klessen, R.S. 2004, Rev. Mod. Phys. 76, 125

Motte, F., André, P. \& Neri, R. 1998, A\&A 336, 150 - MAN98

Motte, F., André, P. \& Ward-Thompson, D. \& Bontemps, S. 2001, A\&A 372, L41

Mouschovias, T.M. \& Ciolek, G.E. 1999, in: C.J. Lada \& N.D. Kylafis (eds.), The Origin of Stars and Planetary Systems (Klewer), p. 305

Myers, P.C. 1998, ApJL 496, L109

Nutter, D., Ward-Thompson, D. \& André, P. 2006, MNRAS 368, 1833 
Onishi, T., Mizuno, A., Kawamura, A., Tachihara, K. \& Fukui, Y. 2002, ApJ 575, 950

Padoan, P. \& Nordlund, A. 2002, ApJ 576, 870

Palouš, J., Ehlerova, S. \& Wünsch, R. 2003, ApESSS 284, 873

Peretto, N., André, Ph. \& Belloche, A., 2006, A\& A 445, 979

Pilbratt, G. 2005, in: A. Wilson (ed.), The Dusty and Molecular Universe: A Prelude to Herschel and ALMA (ESA-SP), 577, p. 3

Shu, F.H., Adams, F.C. \& Lizano, S. 1987, ARAA 25, 23

Shu, F.H., Li, Z.-Y. \& Allen, A. 2004, ApJ 601, 930

Stanke, T, Smith, M.D., Gredel, R. \& Khanzadyan, T. 2006, A\&A 447, 609

Starck, J.L., Murtagh, F. \& Bijaoui, A. 1998, Image Processing and Data Analysis: The Multiscale Approach (Cambridge: Cambridge University)

Tafalla, M., Myers, P.C., Caselli, P., Walmsley, C.M. \& Comito, C. 2002, ApJ 569, 815

Testi, L. \& Sargent, A.I. 1998, ApJL 508, L91

Ward-Thompson, D., André, P., Crutcher, R. et al. 2006, in: B. Reipurth, D. Jewitt \& K. Keil (eds.), Protostars 83 Planets $V$, in press

Ward-Thompson, D., Scott, P.F., Hills \& R.E., André, P. 1994, MNRAS 268, 276

Wootten, A. 2001, Science with the Atacama Large Millimeter Array (ASP-CS), vol. 235

\section{Discussion}

ELMEGREEN: You have sampled several regions now where the cloud masses differ and I wonder if the turnover mass, which might be identified with $M_{J}$, varies in a systematic way?

ANDRE: A fairly large number of regions have indeed been surveyed in the (sub)millimeter continuum by now, and a possible correlation between the break point in the core mass spectrum and parent cloud density or Jeans mass is an effect we have looked for. The most obvious correlation, however, is not with cloud density but with cloud distance, suggesting the break may be partially due to incompleteness at the low-mass end of the core mass spectrum. For instance, Reid \& Wilson (2005, 2006) recently surveyed massive star-forming regions such as M17 at $d=1.6 \mathrm{kpc}$ and found a turnover at $\sim 4 \mathrm{M}_{\odot}$ instead of our values of $\sim 0.3 \mathrm{M}_{\odot}$ in $\rho \mathrm{Oph}$ and $\sim 0.9 \mathrm{M}_{\odot}$ in Orion. On the other hand, the high-mass end of the core mass spectrum seems fairly robust.

MACLOW: How do your results compare to the much shallower spectrum found by Velusamy et al. in a poster at this meeting?

ANDRE: I must admit that I didn't know the study by Velusamy et al.you are referring to. One difference is that they observed at $\lambda=350 \mu \mathrm{m}$, closer to the peak of the core SEDs, i.e., at a wavelength where temperature effects become important. Our observations were done at $\lambda=1.2 \mathrm{~mm}$ or $\lambda=800 \mu \mathrm{m}$, i.e., in the Rayleigh-Jeans regime of the SEDs where in principle we have a more direct proportionality between measured flux and core mass. My other comment is that one has to be very careful when extracting/identifying potential prestellar cores in sub-millimeter star continuum maps. Our team has been using a multi-resolution wavelet analysis to extract starless cloud fragments seen on the same spatial scales as prestellar envelopes observed on the same maps. This is important because if one counts larger structures also visible in our maps as cores, then a flatter mass spectrum is obtained, but these larger structures are too big to qualify as potential progenitors to individual stars or systems (see Motte et al. 1998 and Motte \& André 2000 for details).

VELUSAMY: We used 51 cores observed in our $350 \mu \mathrm{m}$ survey of quiescent cores in Orion region about 30 arcmin to the north and south of Orion KL. They have a power law 
mass function with slope $\alpha=0.85 \pm 0.21$ which is significantly flatter. I would like to add a note of caution interpreting the multiple slopes in the cumulative mass spectra you showed. We find use of cumulative mass functions can erroneously suggest multiple power law indices particularly if core mass distribution is characterized by a power law index $\sim-1$ (Discussed in poster S237-230).

ANDRE: Thank you for pointing your new observations of Orion which I was unaware of. The reason for the difference in the derived core mass spectrum may possibly come from the difference in wavelength $(350 \mu \mathrm{m}$ more sensitive to temperature variations than $850 \mu \mathrm{m}$ or $1.2 \mathrm{~mm}$ ) or a difference in the method used to identify the cores from the maps. Concerning your note of caution, I am well aware that the cumulative mass function and the differential mass function both have advantages and disadvantages. I only showed cumulative plots for the sake of brevity in my talk but the core mass function originally published in our $\rho$ Oph paper (Motte et al. 1998 A\&A) was differential (dN/dM) and was entirely consistent with the broken power law core mass spectrum following the Salpeter slope at the high-mass end. 